\title{
EL DERECHO DE HUELGA EN LA ERA DIGITAL: EL ESQUIROLAJE TECNOLÓGICO
}

\author{
The right to strike in the digital age: technological \\ strikebreaking
}

\author{
Beatriz Sánchez-Girón Martínez \\ Graduada en Derecho y Relaciones Laborales por la UCM y estudiante de máster en la \\ $\mathrm{UC} 3 \mathrm{M}$
}

http://dx.doi.org/10.18543/ed-69(1)-2021pp85-93

Recibido: 04.02.2021

Aceptado: 18.06 .2021

\section{Resumen}

Es de aceptación generalizada que las nuevas tecnologías y la digitalización están cambiando el marco de las relaciones laborales. El derecho fundamental de huelga, un derecho peculiar en cuanto a su reconocimiento constitucional y su escasa regulación, también se ha visto afectado por estos cambios. Las nuevas herramientas proveen al empresario de una mayor cantidad de instrumentos para controlar la organización empresarial y al propio trabajador. En este contexto garantizar el reconocimiento y ejercicio de los derechos fundamentales se hace imprescindible a la vez que complicado. Prueba de ello es el caso del esquirolaje tecnológico: si bien el esquirolaje tradicional ha sido prohibido en la medida en que atenta contra el fin de la huelga, el esquirolaje tecnológico encuentra justificación por no estar referido a trabajadores, si no a elementos tecnológicos. Atendiendo a casos concretos, los tribunales han dado respuestas a los problemas que origina el esquirolaje tecnológico apoyándose en una ponderación entre el ejercicio de un derecho fundamental y el poder organizativo empresarial. El poder del empresario ha crecido frente a las huelgas, lo que hace que se requiera un esfuerzo legislativo moderno para seguir protegiendo el derecho de huelga. 


\title{
Palabras clave
}

huelga, esquirolaje tecnológico, poder empresarial, derecho fundamental.

\begin{abstract}
It is said that the technology and the digitalisation are changing the labour relation's framework. The fundamental principle of the right to strike, unique because although it is enshrined in the Constitution, its legal development is slight, has also been affected by these transformations. Due to the new tools, employers could have a higher control of the production and of the workers. In this context, to guarantee the recognition and effective exercise of the fundamental rights is an essential requirement, but complex as well. Strike-breaking using technology is an example of this: generally speaking, traditional strike-breaking has been banned, however technological strike-breaking could be more easily justified because it is nof affecting workers. The case law shows how the Courts have weight the enjoyment of the fundamental right and the business powers in order to ascertain if the strikes are proportional. The power of the employer has grown, especially in the control of strikes, which means that a modern legislative effort is required to continue protecting the right to strike
\end{abstract}

\section{Keywords}

strike, strike-breaking, business power, fundamental right. 
SUMARIO: I. INTRODUCCIÓN: EL NUEVO ESCENARIO DE LA HUELGA. II. EL ESQUIROLAJE: ESPECIAL MENCIÓN AL ESQUIROLAJE TECNOLÓGICO. III. El ESQUiROLAJE TECNOLÓGICO EN LA JURISPRUDENCIA. IV. CONCLUSIONES. V. BIBLIOGRAFÍA.

\section{INTRODUCCIÓN: EL NUEVO ESCENARIO DE LA HUELGA}

La huelga es, por excelencia la medida de conflicto colectivo de los trabajadores, y goza de reconocimiento constitucional porque es un pilar básico en un Estado Social Democrático (MERCADER). Desde finales del siglo XVIII, la huelga, ha sido utilizada por los trabajadores como instrumento de reivindicación ante su empobrecimiento generalizado a consecuencia de la revolución industrial. No obstante, ha recorrido un tortuoso camino, que comienza con su penalización y tipificación como delito, hasta que finalmente se ha reconocido como derecho fundamental. La historia de la huelga exige prestar atención al pasado, a las circunstancias políticas, sociales y económicas de cada momento, y como no podría ser menos, hoy el uso de las nuevas tecnologías también hace que su ejercicio sea muy diferente a lo que ha sido en épocas anteriores.

La Constitución en su artículo 28.2 reconoce de una manera genérica el derecho a la huelga de los trabajadores, dejando su regulación en manos de una Ley Orgánica aún todavía hoy inexistente. De la doctrina académica y de la jurisprudencia (STC 11/1981) podemos afirmar que la huelga consiste en la interrupción de la prestación de trabajo pactada y coordinada colectivamente por los trabajadores por cuenta ajena, de ahí que sea un derecho de titularidad colectiva, aunque sea individual la decisión de cada uno de unirse o no a la huelga, para la defensa de sus intereses, ocasionando perjuicio a la empresa para que ésta ceda ante las presiones y reconozca las peticiones de estos trabajadores. Gracias al RDLRT (RDL 17/19777, de 4 de marzo sobre relaciones de trabajo) y a la jurisprudencia, especialmente las apreciaciones realizadas del pronunciamiento STC 11/1981, es posible aproximarse hacia un completo contenido jurídico del derecho de huelga con una definición, titularidad, condiciones de su ejercicio y consecuencias del mismo.

La introducción de las nuevas tecnologías en la empresa ha cambiado, entre otros muchos aspectos, la forma de prestación del trabajo pues además de mejorar la eficiencia, ha permitido que el empresario pueda someter a sus trabajadores a los intereses productivos en cualquier lugar y momento, incluso fuera del espacio y tiempo de trabajo, es el empresario panóptico, según el Prof. Mercader. La digitalización de la empresa incrementa el poder de dirección y control empresarial y, en consecuencia, coloca al trabajador en una posición de mayor vulnerabilidad. El uso de dispositivos tecnológicos 
para desempeñar las tareas ha hecho que, frecuentemente, el trabajador acabe siendo sustituido por ellos. Precisamente por estas razones, la huelga no se ha mostrado ajena a la nueva realidad y su regulación jurídica ha quedado obsoleta. Son mucha las voces que alertan de que la era digital pone en difícil protección los derechos de los trabajadores, sin ir más lejos la ONU ha defendido que «es fundamental que estas personas puedan disfrutar de todos sus derechos humanos, entre otros el derecho a la afiliación sindical y a la huelga. En determinados casos, el ejercicio de esos derechos puede poner coto a los excesos de las empresas $\left.{ }^{1}.\right\rangle$

Llegados a este punto, es imprescindible hacerse preguntas: ¿Qué papel juega la huelga si los puestos de trabajo están más deshumanizados que nunca? ¿Qué perjuicio empresarial provocará la ausencia de trabajadores si las tareas están mecanizadas, digitalizadas y automatizadas? ¿Qué sentido tiene la huelga si fácilmente los trabajadores pueden ser sustituidos por herramientas tecnológicas?

\section{EL ESQUIROLAJE: ESPECIAL MENCIÓN AL ESQUIROLAJE TECNOLÓGICO}

Los límites y condiciones del derecho de huelga han sido trazados por el RDLRT y por la jurisprudencia. Por un lado, los trabajadores se prohíben las huelgas ilegales (art.11 RDLRT) y huelgas abusivas (art. 7.2 RDLRT), lo que se resume en que éstas deben guardar proporcionalidad entre el interés defendido y el perjuicio causado, pues lo daños huelguísticos siempre deben ser razonables (STC 123/1992). En el lado opuesto, si nos situamos en el lado empresarial, el mismo RDL en su artículo sexto indica que la sustitución de trabajadores que acuden a la huelga por trabajadores ajenos a la empresa no está permitida, es el llamado esquirolaje externo. La doctrina jurisprudencial ha señalado también la ílicitud del esquirolaje interno, la sustitución de trabajadores en huelga ahora por trabajadores de la empresa encargándoles tareas que no les corresponderían trasladándoles de su centro de trabajo. El esquirolaje vulnera el derecho de huelga en tanto que lo vacía de su contenido, aminorando la presión pretendida sobre el empresario. De la misma manera, el esquirolaje interno podría suponer un ejercicio abusivo de los poderes empresariales. Primero, si se trata de un cambio de las funciones de los trabajadores podría desbordar los supuestos del ius variandi empresarial, por imponer modificaciones de las condiciones de trabajo sin causa alguna. Recordemos que para realizar un cambio de funciones fuera del grupo

\section{Derechos humanos en la era digital. ¿Pueden marcar la diferencia?}

Discurso programático de Michelle Bachelet, Alta Comisionada de las Naciones Unidas para los Derechos Humanos. Japan Society, Nueva York, 17 de octubre de 2019 
profesional o un traslado siempre que sea temporal, es necesario que existan razones técnicas y organizativas para ello (art. $39 \mathrm{ET}$ ) donde no cabría el paliar los efectos de la huelga; y que, por otro lado, la modificación sustancial de las condiciones de trabajo exige un procedimiento regulado en el art. 41 ET. En segundo lugar, si lo que se pretende es un cambio de centro de trabajo, también exige fundadas razones económicas, técnicas, organizativas o productivas.

Como hemos visto, la era digital y tecnológica imponen nuevos retos a la huelga que tradicionalmente se conocía, y el esquirolaje tecnológico, tema central de las siguientes páginas, es uno de ellos. Brevemente, el Tribunal Supremo lo define como «la sustitución de medios humanos por medios mecánicos y automáticos durante la huelga» (STS de 5 de diciembre de 2012)». Es un ejemplo más de cómo los avances tecnológicos originan problemas para los cuales no existe todavía previsión en la regulación normativa, obligando a una modernización del Derecho del Trabajo que ya llega tarde. De hecho, la Profesora Gordillo ha dicho, textualmente que existe «obsolescencia del derecho de huelga en nuestro ordenamiento jurídico». Son nuestros Tribunales quienes tienen que hacer una labor de aplicación e interpretación para adecuar la norma a las nuevas realidades de este mundo tan cambiante.

El establecimiento de los límites en el ejercicio del esquirolaje tecnológico además ha puesto sobre la mesa dos interesantes debates: uno relacionado con el derecho a la libertad empresarial y otro con el poder organizativo empresarial (ius variandi). Las nuevas tecnologías están amenazando los puestos de trabajo porque el empresario está sustituyendo el trabajo humano por máquinas en aras de aumentar la productividad y la eficacia; algo perfectamente posible por el derecho a la libertad de empresa (art $38 \mathrm{CE}$ ). En el caso del esquirolaje tecnológico el objetivo perseguido es sustancialmente diferente: ahora la sustitución de trabajadores se hará de manera temporal, y no definitiva, con el objetivo de evitar los perjuicios que una huelga pudiera ocasionar. En consecuencia, se plantea si podría o no quedar amparado por la libertad de empresa, pues lo que realmente se está tratando de conseguir es una aminoración del impacto del fenómeno huelguístico, práctica que, como ya hemos visto, la jurisprudencia ha considerado ilícita. No obstante, el derecho de huelga no es un derecho de resultado, recuerda el TSJ de Madrid (STSJ Madrid, 23 de julio de 2012), así que «es legítimo, por tanto, que los empresarios traten de atenuar las consecuencias de la huelga mediante aquellos medios de los que dispongan, y siempre que ello se realice mediante conductas lícitas». Fijar los límites en el uso de los medios tecnológicos para no anular el derecho de huelga es una tarea compleja a la que se han enfrentado nuestros tribunales sin tener un criterio armonizado entre los Jueces y Magistrados, como veremos a continuación. 
En segundo lugar, en el caso del esquirolaje tradicional quedaba claro que la asignación a los trabajadores internos de tareas diferentes a las pactadas en su contrato de trabajo constituía una modificación sustancial de las condiciones de trabajo y por lo tanto era una extralimitación del ius variandi del empresario. Cuando nos referimos al esquirolaje tecnológico realmente no se está produciendo ninguna alteración en las condiciones contractuales de trabajo, sino que estamos frente a meras decisiones empresariales organizativas (TASCÓN LÓPEZ).

\section{EL ESQUIROLAJE TECNOLÓGICO EN LA JURISPRUDENCIA}

Como es lógico, resulta más fácil sustituir la labor de los trabajadores en empresas tecnológicas que en aquellas donde el factor humano es imprescindible. El sector que por excelencia ha sufrido las consecuencias del esquirolaje tecnológico, como veremos a continuación, es el audiovisual. La respuesta jurisprudencial a este reciente fenómeno, lejos de ser homogénea, se ha ido conformando a lo largo del tiempo, y la postura de nuestros Tribunales, Supremo y Constitucional hoy no es la misma que la que se adoptó inicialmente. Los pronunciamientos son producto de unas concretas circunstancias que dificultan la construcción de una doctrina general y unitaria.

En un primer momento se decretó la licitud del esquirolaje tecnológico por el TS estableciendo una relación directa el esquirolaje interno: si la empresa emplea los medios técnicos que habitualmente dispone y no ha tenido que adquirir nuevos, y ha empleado a trabajadores con los que ya contaba sin tener que contratar nuevos, no se lesiona el derecho de huelga (STS de 11 de junio de 2012). En este caso, la televisión pública vasca emitió una programación automáticamente pregrabada durante una huelga general. El fallo de esta sentencia viene acompañado de un voto particular incidiendo en que esta práctica sí puede limitar la trascendencia de la huelga en este tipo de empresas y no debería ser legal (Magistrado Excmo. Sr. D. Manuel Ramón Alarcón en la STS 11 de junio de 2012).

Sin embargo, poco tiempo después se planteó un problema muy similar y el TS cambió totalmente su parecer, explicando que se está reduciendo la repercusión la huelga si se emite programación pregrabada, respondiendo así a las críticas que se habían expuesto en el voto particular de la sentencia anterior. En la STS de 5 de diciembre de 2012 se dice que se lesiona el derecho de huelga cuando se realizan actividades más allá de lo decretado como servicio mínimo o «cuando el objetivo sea impedir la eficacia del derecho de huelga» y ésta además de por medios humanos «sea por medio mecánicos o tecnológicos» porque «priva materialmente a los trabajadores de su derecho fundamental, vaciando de su contenido esencial el derecho de huelga». 
El TC se pronunció con relación la licitud del esquirolaje tecnológico en la STC 17/2017 de 2 febrero, única en esta materia. Se plantea un recurso de amparo alegando una vulneración del derecho de huelga por el sindicato CGT en Telemadrid, con ocasión de una huelga general en la que dicha cadena de televisión emitió un partido de fútbol. CGT entiende que la se saboteó así la huelga quedando frustrada su finalidad reivindicativa. El TC responde con una interesante matización, explicando que la utilización de medios con los que dispone la empresa, aunque no sean lo que emplea habitualmente es lícita. En este caso, las funciones de los trabajadores que no secundan la huelga no son diferentes a las que vienen desarrollando y por lo tanto no hay esquirolaje interno. De la misma manera, tampoco hay esquirolaje tecnológico porque, aunque los medios que se emplearon para la emisión del partido de fútbol solo se requieren en situaciones excepcionales, son medios técnicos con los que ya contaba la empresa.

En relación con este asunto, el Magistrado Excmo. Sr. D. Fernando Valdés Dal-Ré emite un voto particular en el que muestra especialmente duro con el fallo del TC, a quien acusa de «no garantizar una protección efectiva de la integridad de los derechos fundamentales» pues la aplicación del artículo $28 \mathrm{CE}$, relativo al derecho de huelga, s hace con una perspectiva limitativa. Acusa al TC de simplificar los hechos de tal manera que no encuentra la vulneración del derecho de huelga que a sus ojos es evidente. Exige, en una correcta apreciación en mi opinión acertada, que este Tribunal se mantenga sensible y abierto a los cambios tecnológicos y los tenga en cuenta para esbozar su la protección que otorga a los derechos fundamentales.

Las cuestiones relativas a este género no se agotan aquí. Salvo error por mi parte, el último pronunciamiento del TS es de hace pocos meses, es en un proceso de unificación de doctrina que, aunque rechazó, son evidencia de que la problemática en torno al esquirolaje tecnológico está a la orden del día (TS Auto de 8 de septiembre de 2020).

Finalmente, se hace obligatorio considerar que supone la actitud adoptada por nuestros tribunales. Cuando se alega una vulneración del derecho fundamental de la huelga, el procedimiento exige una inversión de la carga de prueba: mediante indicios razonables los trabajadores demandantes alegan la vulneración y, es la parte demandante quien tiene que probar que su actuación obedece a fines productivos alejas de la pretendida vulneración del derecho de huelga. La falta de prohibición expresa del esquirolaje interno dejan un amplio margen de actuación al empresario, pues actos que podrían considerarse lesivos ahora han encontrado justificación. Si la empresa emplea sus propios medios y a sus trabajadores, igual que no se puede considerar como una práctica ilícita de esquirolaje interno, tampoco cabe hacerlo como esquirolaje tecnológico. En definitiva, la libertad de empresa parece prevalecer sobre el derecho fundamental de huelga. 
Cuestión diferente que para terminar estas páginas hay que mencionar por ser una consecuencia típica de las empresas actuales es qué ocurre con la huelga y las redes de empresas o empresas descentralizadas. Cuando nos encontramos con grupos de empresas que tienen a su vez diferentes empresas, o centros de trabajo, o empresas que han subcontratado sus actividades con otras, el ejercicio de huelga se hace aún más difícil. En estos casos, el impacto de la huelga es mínimo porque, bien la empresa cuenta con otros centros donde continuar la producción, bien se trata de un entramado de empresas conectadas que cuentan con pocos trabajadores y cuyas tareas son fácilmente sustituibles. El TS (STS 3 de octubre de 2018) si ha catalogado como esquirolaje ilícito acudir a otra empresa contratista para sustituir las actividades laborales de una contrata que está en huelga. Sin embargo, lo que se pone de manifiesto es que la configuración del derecho de huelga tradicional no se adapta a las «nuevas realidades empresariales», en palabras del profesor Goerlich.

\section{IV.CONCLUSIONES}

La huelga ha sido y sigue siendo uno de los instrumentos más valiosos con los que los trabajadores cuentan para defender sus derechos y lograr mejoras en sus condiciones laborales. Su regulación hoy continúa siendo escasa, sujeta las decisiones jurisprudenciales que fijan los límites de su ejercicio y que deben ser adaptadas a la realidad del momento.

La introducción de las nuevas tecnologías ha cambiado el desarrollo de las relaciones laborales. El esquirolaje tecnológico es una nueva herramienta empresarial que puede reducir el éxito de las huelgas y dificultar su ejercicio. Por ello, el derecho de huelga no puede ser ajeno a este fenómeno para el que se necesita una respuesta jurídica porque con el paso del tiempo es muy probable que los problemas que hoy se plantean crezcan. Hasta la fecha, la doctrina constitucional ha aceptado el esquirolaje tecnológico interno, pero no se ha pronunciado acerca del esquirolaje tecnologico externo.

Es importante reforzar el derecho de huelga para que continúe resultando útil como arma de defensa de los intereses de trabajadores. Esto requiere no ignorara la realidad que nos brida el avance tecnológico que, aunque provoca importantes y positivos cambios productivos también aumenta el poder empresarial de manera abusiva respecto de los intereses de los trabajadores. Las respuestas de nuestros tribunales han sido claramente restrictivas y desvirtúan el contenido de la huelga. La doctrina general ha realizado una ponderación entre los poderes empresariales y los derechos fundamentales, apelando a la proporcionalidad en el derecho de huelga. Por ello precisamente, a mi juicio, estimo que la protección de este derecho pasa necesariamente por reforzar los controles empresariales. La definición de esquirolaje 
interno y externo necesita de una relectura adaptada a cada caso concreto que tenga como bandera la protección de la eficacia del derecho de huelga como derecho fundamental amparado por nuestra carta magna.

\section{BIBLIOGRAFÍA}

Cordero Gordillo, Vanessa, La sustitución de los trabajadores huelguistas por medios tecnológicos. Lex Social: revista jurídica de los Derechos Sociales, vol. $9, \mathrm{n}^{\mathrm{o}} 1.2019$.

Goerlich Peset, José María, Ejercicio del derecho de huelga en el contexto de la descentralización productiva. XXVII Congreso AEDTSS. Santiago de Compostela, 2018.

Montoya Melgar, Alfredo, Derecho del trabajo $\left(37^{\mathrm{a}}\right)$. Tecnos, Madrid, 2016.

MerCader Uguina, Jesús R. Derechos fundamentales de los trabajadores y nuevas tecnologías: ¿hacía una empresa panóptica?», Tirant lo blanch, Valencia, 2017.

Mercader Uguina, Jesús R., de la Puebla Pinilla, Ana, Gómez Abelleira, Francisco Javier, Lecciones de derecho del trabajo (9a, Ser. Manuales). Tirant Lo Blanch, Valencia, 2017.

Tascón López, Rodrigo, \& Fernández Domínguez, José Julio, El esquirolaje tecnológico, Aranzadi-Thomson Reuters, Madrid, 2018.

TosCANI GIMÉNEZ, Daniel, Observatorio temático de jurisprudencia La prohibición de esquirolaje durante la huelga con especial mención al esquirolaje tecnológico. Trabajo y Derecho, $\mathrm{n}^{\circ}$ 30, 2017. 


\title{
EL DERECHO DE HUELGA EN LA ERA DIGITAL: EL ESQUIROLAJE TECNOLÓGICO
}

\author{
The right to strike in the digital age: technological \\ strikebreaking
}

\author{
Beatriz Sánchez-Girón Martínez \\ Graduada en Derecho y Relaciones Laborales por la UCM y estudiante de máster en la \\ $\mathrm{UC} 3 \mathrm{M}$
}

http://dx.doi.org/10.18543/ed-69(1)-2021pp85-93

\section{Copyright}

Estudios de Deusto es una revista de acceso abierto, lo que significa que es de libre acceso en su integridad. Se permite su lectura, la búsqueda, descarga, distribución y reutilización legal en cualquier tipo de soporte sólo para fines no comerciales, sin la previa autorización del editor o el autor, siempre que la obra original sea debidamente citada y cualquier cambio en el original esté claramente indicado

Estudios de Deusto is an Open Access journal which means that it is free for full access, reading, search, download, distribution, and lawful reuse in any medium only for non-commercial purposes, without prior permission from the Publisher or the author; provided the original work is properly cited and any changes to the original are clearly indicated. 